\title{
EUS-Guided Drainage of Liver Abscesses: Ultra Uncertain or Sound Practice?
}

\author{
Maria Chiara Petrone ${ }^{1}$ Paolo Giorgio Arcidiacono ${ }^{1}$
}

Published online: 19 October 2015

(C) Springer Science+Business Media New York 2015

\section{Introduction and Background}

Liver abscesses, which are collections of infected necrotic tissue within the hepatic parenchyma, as the most common intra-abdominal abscess have a reported incidence of 8-20 cases/100,000 hospitalized patients/year in the USA [1]. Abscesses, which are frequently attributed to polymicrobial infections (e.g., Klebsiella species) and less frequently by Entamoeba, are typically associated with biliary tree infection due to benign or malignant obstruction, and rarely as an extension of intra-abdominal infections (e.g., appendicitis) or via hematogenous spread due to septicemia. Recent diagnostic and therapeutic advances that have facilitated early diagnosis, that have supported the extensive use of specific antibiotics, that have furthered the development of radiologic aspiration or drainage techniques, and have driven the evolution of modern liver surgery, have substantially modified the clinical course of liver abscesses. In a recent meta-analysis [2], percutaneous drainage (PCD) of liver abscesses was reported to be superior to percutaneous aspiration alone.

In the last decade, endosonography (EUS)-guided drainage has become the first-line treatment for pancreatic and peri-pancreatic fluid collections, regardless of the abscess contents. Much recent data support the efficacy and safety of EUS-guided procedures for diagnostic and therapeutic access to the biliary tree in cases of failure of endoscopic retrograde cholangio-pancreatography (ERCP) drainage

Paolo Giorgio Arcidiacono

arcidiacono.paologiorgio@hsr.it

1 Pancreato-Biliary Endoscopy and Endosonography Division, San Raffaele Scientific Institute, Vita Salute San Raffaele University, Milan, Italy
[3]. The availability of fully covered, self-expandable metal (mesh) stents (FCSEMS) specifically designed for these interventions has greatly increased the numbers of EUS-guided drainage procedures performed globally. Due to these improvements, including the ability to easily target the liver under EUS guidance, individual case reports-and now case series-are reporting safe and effective echoendoscopic drainage of hepatic abscesses by means of plastic stents or with preference to standard or lumen-apposing FCSEMS.

\section{Comparison of EUS-AD with PCD}

Until now, no study has compared EUS-guided abscess drainage (EUS-AD) to PCD in the treatment of liver abscesses. In this issue of Digestive Diseases and Sciences, Ogura and colleagues [4] evaluated the safety and feasibility of EUS-guided drainage of hepatic abscess using FCSEMS, comparing the clinical outcomes of EUS-AD versus $P C D$ in a retrospective study in which EUS-AD was performed in patients considered unfit for PCD (i.e., those with ascites, with dementia, or in whom a safe puncture tract was not identified). Seventy percent of the abscesses treated by EUS-AD were located in the left liver lobe; the right lobe abscesses were predominantly managed by PCD. EUS-AD by FCSEMS was safe and effective with $100 \%$ technical success, no adverse events, and a statistically significant decrease in median hospital stay (21 vs 41 days). On the basis of these data, the authors concluded that EUS-AD could become the first-line treatment for hepatic abscesses, particularly those located in the left liver lobe.

As a first step toward a new indication for therapeutic EUS, we congratulate the authors for performing this 
demanding study, which presents an opportunity to highlight the advantages of EUS-guided treatment, but also raises questions that are far from being answered. The pros of the procedure have been well covered in the paper: EUS$\mathrm{AD}$ is safe and effective for treating liver abscesses, and the use of large diameter (e.g., $10 \mathrm{~mm}$ ) FCSEMS compared with the $\sim 3-\mathrm{mm}$ plastic stents employed for PCD probably explains the significant shortening of hospitalization length and possibly could improve the quality of life, since internal FCSEMS avoid the risk of accidental or intentional removal of percutaneous drains, especially by confused, agitated patients. What are the bones of contention that this study unearthed? First, while this is a retrospective study performed in a population with a high prevalence of left lobe abscesses, epidemiologic studies report a right hepatic lobe predominance of hepatic abscesses (typically 70 vs. $30 \%)[5,6]$. Second, due to anatomic limitations, EUS cannot visualize the entire hepatic parenchyma, particularly the distal segments of the right liver lobe (e.g., liver segments VI, VII, and VIII). Since visualization of the right lobe segments is challenging and instrumentation risky, with an enhanced possibility of duodenal perforation and other complications even in the hands of a skilled endosonographer, EUS-guided drainage of right-sided liver abscesses is currently difficult to justify. Third, since current data only support the use of EUS-AD-guided drainage for one abscess at a time and since nearly $20 \%$ of liver abscesses are multiple, EUD-AD should not be used for multiple abscess drainage.

\section{Technical Skills and Costs}

PCD is a simple procedure that can be performed at the bedside (if necessary) in any hospital with interventional radiology facilities using relatively inexpensive tools (i.e., needles, guide wires, plastic double pigtail stents). PCD can be performed for single and multiple abscesses located in the right or the left lobe, with technical success approaching $100 \%$ and clinical success close to $85 \%$ [7]. In comparison, EUS-AD is a technically demanding procedure that can be attempted only in major referral centers by echoendoscopists with specific training and experience. The difficulty of EUS-guided drainage is compounded by the circumstance that, unlike the pancreas, the liver does not firmly abut the stomach. Moreover, the need for a series of steps to achieve final deployment of the stent increases the risk of failure. The use of standard dilators to increase the size of the tract through the parenchyma has been superseded by the introduction of a 2.2-mm-diameter "cystotome" that creates a tract ready for stent deployment. The availability of a lumen-apposing FCSEMS with single-step deployment facilitates procedure completion using only one device under EUS control and without fluoroscopy. Although FCSEMS are increasingly used for pancreatic pseudocyst drainage, they may not always be suitable for treating remotely located abscess cavities. The use of partially covered, self-expanding metal mesh stents specially designed for hepatogastrostomy $[8,9]$ will likely also reduce technical difficulties. Since the presence of ascites increases the space between the gastric wall and the hepatic parenchyma and also increases hepatic mobility, the authors' suggestion that the presence of ascites may be an indication for EUS-AD instead of PCD has to be carefully evaluated.

Considering the cost of the instrumentation, the required devices (EUS needle, guide wire, balloon dilator, FCSEMS), and the support personnel involved, usually including an anesthesiologist for general anesthesia with endotracheal intubation, EUS-AD is extremely expensive. In this era of cost containment, a cost/benefit analysis would need to document indirect cost savings such as reduced length of stay that offset increased procedure-related costs.

\section{Long-Term Follow-Up}

The median follow-up reported in the study was nearly 7 months in both groups, with a very low rate of recurrence for the PCD group and no recurrence in the EUS-AD group. The authors suggest that this impressive result with EUS-AD is related to the permanence of the FCSEMS since they are not removed, which might explain their early success, with the authors yet to report longer-term outcomes. Notwithstanding that the majority of liver abscesses occur in patients with benign disease such as common bile duct stones or cholecystitis who are expected to survive many years after successful treatment, FCSEMS are usually placed to palliate malignant biliary strictures in patients with terminal disease and short anticipated survival, often $<1$ year. When used for pancreatic pseudocyst drainage, FCSEMS are usually removed after one or 3 months. Case reports describe short-term mucosal overgrowth involving these stents that hampers their removal. Moreover, occlusion of FCSEMS by food debris can cause fluid collections to reform and become infected. These considerations prompt several questions: First, there were no data regarding the long-term follow-up of the patients, especially those related to abscess recurrence or acute cholangitis years after FCSEMS placement; second, there are no data regarding stent removal as is possible for plastic catheters in PCD and SEMS in pseudocysts. For the second question, the answer is probably no for the partially covered stents and possibly yes for the fully covered ones, but with the potential for major complications such as hemorrhage, bile leakage, and peritonitis. 


\section{Conclusions}

In summary, the authors have presented compelling evidence of the near-term safety and efficacy of EUS-guided drainage of hepatic abscesses compared with the current standard-of-care PCD. Prior to replacing PCD for routine hepatic abscess drainage, many issues need to be addressed, namely long-term results, cost/benefit, ease of removal, and efficacy in select populations such as in patients with ascite, with multiple abscesses, and with right-sided lesions. It is likely that large, carefully designed randomized clinical trials will be needed to provide the data needed to fully answer these questions before this technique becomes widely adopted by endosonographers.

\section{References}

1. Johannsen EC, Sifri CD, Madoff LC. Pyogenic liver abscesses. Infect Dis Clin North Am. 2000;14:547-563.
2. Cai YL, Xiong XZ, Lu J, et al. Percutaneous needle aspiration versus catheter drainage in the management of liver abscess: a systematic review and meta-analysis. HPB (Oxford). 2015;17:195-201.

3. Singhal S, Changela K, Lane D, et al. Endoscopic ultrasoundguided hepatic and perihepatic abscess drainage: an evolving technique. Therap Adv Gastroenterol. 2014;7:93-98.

4. Ogura T, Masuda D, Saori O, et al. Clinical outcome of endoscopic ultrasound-guided liver abscess drainage using self-expandable covered metallic stent (with video). Dig Dis Sci. 2015 (Epub ahead of print). doi:10.1007/s10620-015-3841-3.

5. Lee KT, Wong SR, Sheen PC. Pyogenic liver abscess: an audit of 10 years' experience and analysis of risk factors. Dig Surg. 2001;18:459-466.

6. Alvarez Pérez JA, González JJ, Baldonedo RF, et al. Clinical course, treatment, and multivariate analysis of risk factors for pyogenic liver abscess. Am J Surg. 2001;181:177-186.

7. Zerem E, Omerovic S, Kunosic S. Sonographically guided percutaneous treatment of liver abscesses in critically Ill patients. J Clin Ultrasound.. 2014;42:527-533.

8. Giovannini M, Bories E, Téllez-Ávila FI. Endoscopic ultrasoundguided bilio-pancreatic drainage. Endosc Ultrasound. 2012;1:119-129.

9. Song TJ, Lee SS, do Park H, et al. Preliminary report on a new hybrid metal stent for EUS-guided biliary drainage (with videos). Gastrointest Endosc. 2014;80:707-711. 